\title{
A second method for inversion of space in classical mechanics
}

\author{
Z. Peykov' ${ }^{1}$ A. Apostolov ${ }^{2}$ \\ University of Architecture, Civil Engineering and Geodesy, Sofia, Bulgaria
}

\begin{abstract}
A method of inversion of space is proposed on the basis of the generally accepted opinion in cosmology for expansion of the Universe as a result of the expansion of space. It is based on the characteristic of space to change its volume as a result of expansion or contraction. The mathematical method is based on Hubble's law describing the escape of galaxies and the expansion of the Universe. The inversion of space replaces its extension with corresponding contraction. Discussion on the proposed method was implemented.
\end{abstract}

Keywords: space, inversion of space

\section{I.INTRODUCTION}

In our previous work, [1] an inversion of space was examined, based on its characteristic a certain direction in it to be defined. In this article, we offer a second way of inversion of space, based on its ability to change its volume.At the beginning of the $20^{\text {th }}$ century, the American astronomer Hubble, exploring the spectrum of distant galaxies in the Universe, discovered the so-called phenomenon redshift of the spectral lines in it $\mathrm{z}=\Delta \lambda / \lambda$, which is proportional to the distance $r$ to a particular galaxy. This is illustrated in Figure 1, taken from [2], where $p_{c}$ is the visible photographic magnitude of the galaxy, proportional to the distance to it. By the middle of $20^{\text {th }}$ century an opinion was imposed among the astronomers that the redshift in the spectrum of galaxies was the result of Doppler's effect, which linked this shift with the radial velocity (along the ray of vision) of the galaxy with respect to the observer, namely:

$$
\mathrm{V}_{\mathrm{r}}=\mathrm{cz}
$$

where $\mathrm{c}$ is the velocity of light.

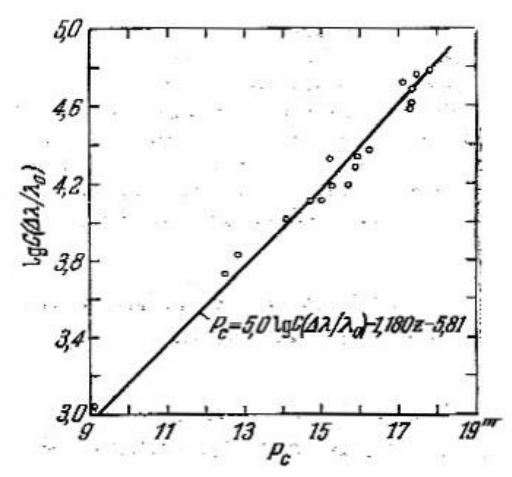

Figure 1: Illustration of the redshift in the spectrum of galaxies depending on the distance to them

From here, Doppler deduced a law that says:

$$
\mathrm{V}_{\mathrm{r}}=\mathrm{Hr}
$$

The radial velocity of receding of each galaxy from the Earth is proportional to the distance to it (the so-called Hubble's law). The constant of proportionality $\mathrm{H}$, called the Hubble's constant, can be determined through observations. Its value for most observations is around $\mathrm{H}=55 \mathrm{~km} / \mathrm{s} . \mathrm{Mpc}$, although that according to latest data its value may be even lower. The physical meaning of Hubble's law is that, on average, the more a galaxy is farther away from the Earth, the faster it moves away from it, i.e. the Universe expands in space proportionally to the distance in it. (There are also opinions that Hubble's law is not due to the effect of Doppler, which, however, are not universally accepted.) 
Using the value of $\mathrm{H}$ and assuming that the speed of expansion of the Universe does not change over the time, we can determine the age of the Universe at a cosmological scale, i.e. the time from its zero dimensions to its current observed dimensions:

$$
\mathrm{t}=1 / \mathrm{H}
$$

Using the above value of $\mathrm{H}$ and considering that: $1 \mathrm{pc}=3,094.10^{16} \mathrm{~m}$ (the distance, below which the radius of the Earth's orbit is seen at an angle of $1 "), \quad 1$ year $==31556736 \mathrm{~s}$, we receive for the age of the Universe: $\mathrm{t}=17,8.10^{9}$ years, which comply with the age of the oldest objects in the galaxies - the globular clusters.

We can also determine the distance at which the velocity of escape of galaxies is close to the velocity of light (the socalled problem with the horizon in cosmology), as the distance to which we can observe the Universe:

$\mathrm{r}_{\mathrm{h}}=\mathrm{c} / \mathrm{H}=5,45.10^{9} \mathrm{pc}=17,8.10^{9}$ light years

Of course, these are rough values that depend on the value of $\mathrm{H}$ and the rate of expansion of the Universe.

\section{METHODS AND MATERIAL}

\section{A. Expansion of the space.}

Without going into the details concerning the cosmological aspects of the expansion of the Universe, its curvature, according to the general theory of relativity and whether it is open, flat or closed, we will note the following. At the beginning of the $21^{\text {st }}$ century, opinions $[3,4]$ were ascertained that the expansion of the Universe was due to the expansion of the space itself, where the Universe is located. Figure 2, taken from [4], shows why there is no centre of expansion of the Universe and why the velocity of expansion is proportional to the distance from a particular point in it.

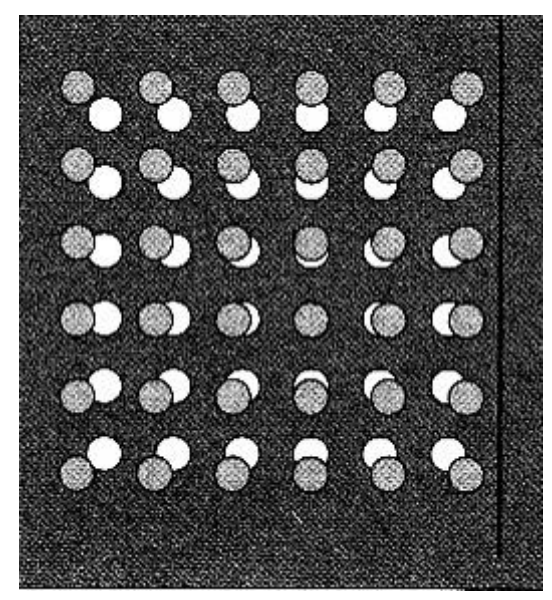

Figure 2: Illustration of the expansion of space in the Universe.

According to these opinions, the space itself in the Universe expands as it continually increases its volume and its expansion is proportional to the distance from a certain point in it. Then Hubble's law will describe not only the escape of galaxies, but the expansion of space itself as well.

Using Hubble's law, we can determine for example the velocity of space expansion at a distance: $\mathrm{r}_{1}=1 \mathrm{~m}$ from a particular point in it:

$$
\mathrm{V}_{\mathrm{r} 1}=\mathrm{Hr}_{1}=17,8 \cdot 10^{-19} \mathrm{~m} / \mathrm{s}
$$

which is practically impossible to be determined through a particular experiment. Even at a distance equal to the radius of our Galaxy: $r_{g}=30.103 \mathrm{pc}$, the velocity of expansion is $\mathrm{V}_{\mathrm{rg}}=1.65 \mathrm{~km} / \mathrm{s}$, which is very difficult to be determined through observations.

\section{B. Second method of inversion of space.}

Using the idea for expanding of space, we will give a second way for its inversion. Under inversion of space, we will understand the replacement of its expansion with a contraction in time, i.e. the inverted space will contract as its contraction is proportional to the distance from a particular point in it. 


\section{International Advanced Research Journal in Science, Engineering and Technology}

Vol. 8, Issue 7, July 2021

\section{DOI: $10.17148 /$ IARJSET.2021.8703}

Mathematically the inverted space will be described by the law of Hubble, as in it the projection of the radial velocity $V_{r}$ will be a negative magnitude:

$$
-\mathrm{V}_{\mathrm{r}}=\mathrm{Hr}
$$

In other words, the highest velocity of contraction will be at the longest distance $\mathrm{r}$ in it. Figure 2 will remain symmetrical, but will now respond to a contraction of space.

\section{III.RESULTS AND DISCUSSION}

1. The specified method of inversion of space is mathematically identical to the method of time inversion described in [5] and consists of changing the sign of time and velocity. This is because here we suppose the movement of the space itself (expansion or contraction) that takes place over the time.

2. This method of inversion of space is valid only if the idea of the expansion of the space itself is true.

3. Here we again, do not concern cosmological questions about the Universe itself - the problem of the horizon, the inflation of the Universe after the Big Bang, its curvature and whether it is open, flat or closed, as well as the presence of gravitational forces in it.

4. The expansion or contraction of space raises the question of its density versus the density of time [6], which we will not discuss here.

5. The "foolish" idea can be assumed that the escape of galaxies in the Universe is due to the very expansion of space [3] as well as the fact that matter itself in the Universe is expanding together with the space that in the ordinary world is practically impossible to be detected experimentally.

6. According to the cosmology the expansion or contraction of the Universe and space is only valid if Hubble's law for redshift is explained through the effect of Doppler due to the movement of galaxies. Other opinions exist as well that the redshift in the spectra is due to the very "aging" of the photons - the reduction of their energy during their movement through the space, but not to the movement of the galaxies themselves, but we will not discuss this here.

\section{IV.CONCLUSION}

In conclusion, we will note that the idea of inversion of space is compatible with the cosmological consideration of the Universe.

\section{REFERENCES}

[1] Z. I. Peykov, A. T. Apostolov, "Inversion of Space in the Classical Kinematics of Material Point”, IJSRST, v. 2, No. 5, p. 309 , 2016.

[2] D. Ya. Martynov, “The course of general astrophysics”, Moscow,Science, 1979. „Курс общей астрофизики“, Д.Я.Мартинов, Москва, Наука, 1979 г.

[3] Lrian Greene, "The fabric of the cosmos: Space, time and the texture of reality", 2004.

[4] L. M. Urauss, "A Universe From Nothing: Why There is Something Rather Than Nothing”, A Division of Simon\& Schuster Inc., 2012.

[5] Z. I. Peykov, T. P. Spiridonov, A. T. Apostolov, "Inversion of Time in the Classical Kinematics of Material Point”, IJSRST, v. 2, No. 5, p. 116, 2016.

[6] Z. I. Peykov, A. T. Apostolov, N. S. Mihailov, "Density of Space and Time in the Classical Kinematics of Material Point", IJSRST, v.2, No. 6, p. $471,2016$. 